\title{
Polarization dependence of the optical absorption of single-walled carbon nanotubes
}

\author{
Yoichi Murakami, Erik Einarsson, Tadao Edamura and Shigeo Maruyama* \\ Department of Mechanical Engineering, The University of Tokyo \\ 7-3-1 Hongo, Bunkyo-ku, Tokyo 113-8656, Japan
}

*Corresponding author: Tel \& Fax: +81-3-5800-6983.

E-mail address: maruyama@photon.t.u-tokyo.ac.jp (S. Maruyama).

\begin{abstract}
Anisotropic optical absorption properties of single-walled carbon nanotubes (SWNTs) are determined from a vertically aligned SWNT film for $0.5-6 \mathrm{eV}$. Absorption peaks at 4.5 and $5.25 \mathrm{eV}$ are found to exhibit remarkable polarization dependence, and have relevance to optical properties of graphite. A method for determining a nematic order parameter for an aligned SWNT film based on the collinear absorption peak at $4.5 \mathrm{eV}$ is presented, followed by the determination of the optical absorption cross-section.
\end{abstract}

PACS numbers: 78.66.Tr, 78.67.Ch 
Single-walled carbon nanotubes (SWNTs) are a new group of carbon material possessing one-dimensional cylindrical geometry formed by rolling-up an $\mathrm{sp}^{2}$-bonded graphene sheet [1]. Their 1D structure and nanoscale diameter gives rise to quantization of the circumferential wave vectors, creating strong divergences in the electronic density of states known as van Hove singularities, which formulate discrete energy levels or "subbands" [2]. The resulting unique properties make SWNTs an important candidate for various electronic and optical applications [3-6]. In particular, since the inter-subband gap energies correspond to infrared to visible light, several innovative optical applications have been proposed [4-6]. The 1D shape of a SWNT would be advantageous in polarization-sensitive optical devices, provided a sound understanding of its anisotropic optical properties.

Despite their significant potential, there have been few experimental studies on the anisotropic optical absorption of SWNTs to provide confirmation to earlier theoretical works in the inter-subband [7] and higher energy regions [8]. So far, such investigations using aligned SWNTs embedded in a polymer matrix [9] and magnetically aligned SWNTs in gel [10] have been reported. However, all the past studies were limited to less than $3.5 \mathrm{eV}$ mainly because of UV absorption by the employed polymers. Investigation of absorption features in the higher energy region containing the $\pi$-plasmon near $5 \mathrm{eV}$ is urgent because they form the baselines in the inter-subband region that are of interest in many optical measurements and applications.

In this Letter, we present anisotropic optical absorption properties of SWNTs for the energy range 0.5 - $6 \mathrm{eV}$ determined by measurements of a vertically aligned SWNT film grown on an optically polished quartz substrate [11]. In addition to the inter-subband region below $3 \mathrm{eV}$, this Letter presents for the first time the remarkable polarization dependence of absorption peaks at 4.5 and $5.25 \mathrm{eV}$. Furthermore, we reveal the relevance of these absorption peaks of SWNTs to the optical properties of graphite. Finally, a method for determining a nematic order parameter of the aligned SWNT film is introduced, followed by determination of optical absorption cross-section of the SWNTs.

The vertically aligned SWNT film was grown on both sides of a quartz substrate supporting Co catalyst [11], using the alcohol CCVD method [12]. The film consists only of SWNTs that are sufficiently clean, i.e. contain virtually no amorphous carbon and no multi-walled carbon nanotubes, as confirmed by resonant Raman scattering (G/D ratio > 25) [11] and high-resolution transmission electron microscopy (HR-TEM) [13]. Measurements of more than 50 SWNTs by HR-TEM revealed an average diameter of $\sim 2.0 \mathrm{~nm}$ with a standard deviation of $\sim 0.4 \mathrm{~nm}$ [13]. Most SWNTs in the film form bundles with a typical diameter of $\sim 15 \mathrm{~nm}$.

For optical absorption measurements, we used a UV-vis-NIR spectrophotometer (Shimadzu UV-3150) with a rotating substrate holder set behind a UV-vis-NIR polarizer. After baseline acquisition, we scanned from $200-2500 \mathrm{~nm}$ at a specified incident angle $(\theta)$ and polarization toward the substrate (s or 
p). By convention, $\theta=0$ means normal to the substrate, and incident light is s-polarized (p-polarized) when its electric field vector is perpendicular (parallel) to the plane of incidence. Reflectance baseline of the bare quartz substrate for $0^{\circ}<\theta<45^{\circ}$ is quite exiguous, contributing to a measured absorbance of less than 0.03 for $\mathrm{p}$ - and 0.06 for s-polarizations, although corrections for this have been made in the following results.

Figure 1 shows polarized absorption spectra from the aligned SWNT film for s- and p-polarized light normalized by the $\cos ^{-1} \theta$ increment of the light path length. The angle $\theta$ was changed from $0^{\circ}$ to $45^{\circ}$ in $7.5^{\circ}$ steps. The inset shows a cross-sectional FE-SEM image of the measured sample, which has a thickness of $2.1 \mu \mathrm{m}$ per side. In the case of s-polarization, dominant absorption by non-collinear dipoles perpendicular to the SWNT axis and weaker collinear dipole absorption arising from disorder in the alignment result in an absorption spectrum independent of $\theta$. The case of p-polarization, on the other hand, shows clear anisotropy, reflecting dominant absorption by collinear dipoles parallel to the SWNT axis. In the lower energy region $(<3 \mathrm{eV}$ ), inter-subband absorption maxima for $\Delta \mu=0$ transitions $(\mu$ denotes the cutting-line index of the 2D Brillouin zone of graphite [2]) are observed. These peaks are the first $(\sim 0.63 \mathrm{eV})$ and second $(\sim 0.93 \mathrm{eV})$ subband gaps in a semiconducting SWNT and the first gap $(\sim 1.45 \mathrm{eV})$ in a metallic SWNT. These energies correspond to a tube diameter of $1.85-1.9 \mathrm{~nm}$ according to recent experimental studies [14], which is consistent with the above TEM measurement.

Most noticeable is the polarization dependence of the peaks at 4.5 and $5.25 \mathrm{eV}$. These peaks have been observed in several studies of randomly oriented SWNT films [15-18] but considerable disagreements are found among the discussions. Kataura et al. [15] attributed the peak at $4.5 \mathrm{eV}$ to $\pi$-plasmon while the $\sim 5.2 \mathrm{eV}$ peak was only seen in the pristine sample containing amorphous carbon. Pichler et al. [16] stated from their EELS measurements on purified SWNTs that the $5.2 \mathrm{eV}$ peak observed at low electron momentum transfer was due to a $\pi$-plasmon along the SWNT axis. Reed and Sarikaya performed EELS on purified SWNTs in a TEM [17], and explained the peaks at 4.2-4.5 eV and $5.2 \mathrm{eV}$ as surface and bulk $\pi$-plasmon excitations, respectively.

Apart from SWNTs, the absorption at $\sim 4.5 \mathrm{eV}$ is commonly observed in graphitic materials [19]. It is known that the imaginary part of the dielectric function for graphite perpendicular to the $c$-axis $\operatorname{Im}\left\{\varepsilon_{\perp}\right\}$ has a maximum at $4.5 \mathrm{eV}$ whereas the maximum of the EELS function in the same direction $\operatorname{Im}\left\{-\varepsilon_{\perp}{ }^{-1}\right\}$ is found at $\sim 7 \mathrm{eV}[20,21]$. These peaks are also confirmed in Ref. 22 in which the crystal local field effect (LFE) was included in the TDLDA calculation. The result matches experimental EELS data for 0 - 40 $\mathrm{eV}$ not only when the momentum transfer $\boldsymbol{q}$ is perpendicular to $c$-axis but also when the deviation of $\boldsymbol{q}$ from that direction is not large [22]. In the direction parallel to the $c$-axis, however, the optical properties of graphite have not yet been fully elucidated because of difficulties in preparing good optical surfaces 
[20,23]. According to EELS data by Venghaus [24], the maximum in $\operatorname{Im}\left\{-\varepsilon_{\|}^{-1}\right\}$ appears at $\sim 5.2 \mathrm{eV}$ $[20,21]$.

From the fact that these two peaks are observed at almost the same positions regardless of the diameter and preparation method of SWNTs [13,15-18], it is deduced that the observed peaks at 4.5 and $5.25 \mathrm{eV}$ in Fig. 1 originate from the optical properties of graphite, corresponding to the maxima in $\operatorname{Im}\left\{\varepsilon_{\perp}\right\}$ (parallel to the SWNT axis) and $\operatorname{Im}\left\{-\varepsilon_{\|}^{-1}\right\}$ (perpendicular to the SWNT axis), respectively. The consistent polarization behavior of the absorption peaks at 4.5 and $5.25 \mathrm{eV}$ can be found in the absorption of a purified SWNT aligned thin-film [25], although unmentioned by the authors. In a practical sense, the magnitude of the 4.5 and $5.25 \mathrm{eV}$ peaks can provide a quantitative index for evaluating the degree of SWNT alignment. More importantly, this optical relevance between SWNTs and graphite above the inter-subband region indicates the possibility of studying the optical properties of graphite by studying SWNTs as arrays of graphene cylinders.

Figure 2(a) shows the lower energy part $(0.5-3 \mathrm{eV})$ of Fig. 1 for p-polarization. At each energy the absorbance increases according to $\sin ^{2} \theta$ and the dotted line denotes the extrapolated spectrum for $\theta=$ $90^{\circ}$. We denote the measured absorbance at $\theta=0^{\circ}$ as $\Lambda_{\perp}$ and that at $\theta=90^{\circ}$ as $\Lambda_{\|}$. The total absorbance $\Lambda_{0}\left(=\Lambda_{\|}+2 \times \Lambda_{\perp}\right)$ is shown as a dashed line. From measured $\Lambda_{\perp}$ and $\Lambda_{\|}$, optical absorption anisotropy $\alpha$ and polarization $\rho$ [26] can be calculated as

$$
\begin{aligned}
& \alpha \equiv \frac{\Lambda_{\|}-\Lambda_{\perp}}{\Lambda_{\|}+2 \Lambda_{\perp}}=\frac{1}{2}\left(3<\cos ^{2} \varphi>-1\right) \\
& \rho \equiv \frac{\Lambda_{\|}-\Lambda_{\perp}}{\Lambda_{\|}+\Lambda_{\perp}}
\end{aligned}
$$

where $\langle\varphi>(0 \leq \varphi \leq \pi / 2)$ denotes an average deviation angle of the SWNT axis from the normal to the substrate. Figure 2b shows $\alpha$ and $\rho$ in this study, where the latter is much improved from $\rho<0.15$ reported in Ref. 10. These values are larger in the lower energy region $(<3 \mathrm{eV})$ because inter-subband absorption parallel to SWNT axis is dominant. At higher energy, the anisotropy decreases because the magnitude of inter-subband absorption is weakened and the baseline of non-collinear dipole absorption becomes noticeable, as confirmed below.

In calculating the order parameter $S$ (which $=\alpha$ if transition dipoles are perfectly collinear) and $\langle\varphi\rangle$, one cannot use Fig. $2 \mathrm{~b}$ because non-collinear dipoles had been included as a disorder in the definition of Eq. 1. To separate the collinear dipole absorption from the spectrum we choose the feature at $4.5 \mathrm{eV}$, as it exhibits the clearest $\theta$ dependence. Based on absorption maxima of either $\operatorname{Im}\{\varepsilon\}$ or $\operatorname{Im}\left\{-\varepsilon^{-1}\right\}$ at $\sim 2.8$, 4.0, 4.4, and $5.2 \mathrm{eV}$ presented by Lee et al. [27], Lorentzian curve fitting was performed for 2.5 - $6 \mathrm{eV}$. 
Figure 3 shows the decomposed spectra obtained by setting four peaks at $2.8,4.0,4.5$, and $5.3 \pm 0.1$ $\mathrm{eV}$. Their widths were fixed at $4.00,1.30,0.98$, and $1.72 \pm 0.02 \mathrm{eV}$, respectively. We have confirmed the absorption spectra of other non-aligned SWNTs with different diameters are also fitted well using the same peak positions and widths [13].

Figure 4a shows the change of each Lorentzian amplitude. The baseline of the non-collinear peak at $5.25 \mathrm{eV}$ may be ambiguous because it locates near the border of the measured range. By fitting the plot for the $4.5 \mathrm{eV}$ peak with $\sin ^{2} \theta, \alpha=S \approx 0.75$ and $\langle\varphi\rangle \approx 24^{\circ}$ are calculated from Eq. 1 . Using molar absorption cross-sections of the SWNT film sample parallel $\left(\eta_{\|}\right)$and perpendicular $\left(\eta_{\perp}\right)$ to the z-axis determined from Fig. 2a [28], and a C atom density of the sample that has been estimated to be $\sim 3.0 \times$ $10^{3} \mathrm{~mol} / \mathrm{m}^{3}$, the bare optical absorption cross-sections of the SWNTs parallel $\left(\sigma_{\|}\right)$and perpendicular $\left(\sigma_{\perp}\right)$ to the SWNT axis can be calculated using the relationships [10]

$$
\begin{aligned}
& \sigma_{\|}=\frac{1}{3}\left\{2 \eta_{\perp}+\eta_{\|}+\frac{2\left(\eta_{\|}-\eta_{\perp}\right)}{S}\right\} \\
& \sigma_{\perp}=\frac{1}{3}\left(2 \eta_{\perp}+\eta_{\|}-\frac{\eta_{\|}-\eta_{\perp}}{S}\right)
\end{aligned}
$$

Figure $4 \mathrm{~b}$ shows $\sigma_{\|}$and $\sigma_{\perp}$ for $0.5-6 \mathrm{eV}$. While $\Delta \mu=0$ inter-subband absorption is seen in $\sigma_{\|}$, it is clear that $\sigma_{\|}$and $\sigma_{\perp}$ have maxima at $\sim 4.5$ and $5.25 \mathrm{eV}$, respectively. It is noted that even in the low energy region $(<3 \mathrm{eV})$, there exists small but non-zero $\sigma_{\perp}$ partially due to an extension of the $\operatorname{Im}\left\{-\varepsilon_{\|}^{-1}\right\}$ $\pi$-plasmon at $5.25 \mathrm{eV}$ into the lower energy region. Further, depolarization effects in a SWNT for cross-polarized light [7] can be imperfect when SWNTs are bundled, leading to $\Delta \mu= \pm 1$ inter-subband transitions [29]. This depolarization effect might also contribute to the enhancement of $\sigma_{\perp}$. In the case of Raman scattering, the scattering by cross-polarized light is suppressed when SWNTs are isolated [30] while it is recognizable with our bundled sample [31]. Therefore, both parallel and perpendicular dipoles to the SWNT axis should be taken into account even in optical measurements for the inter-subband energy region.

In summary, we present anisotropic optical absorption properties of SWNTs for $0.5-6 \mathrm{eV}$, determined from polarized optical absorption measurements of a vertically aligned SWNT film. This is the first report to investigate the absorption features of SWNTs at 4.5 and $5.25 \mathrm{eV}$ and their polarization dependencies. These peaks exhibited very little dispersion $(\sim 0.1 \mathrm{eV})$ on the light polarization, differently from earlier theoretical works including intertube Coulomb interactions [8] in which energies 
of the $\pi$-plasmon peaks remarkably dispersed by the nanotube geometry (i.e. diameter, chiral angle) and the incident light polarization. This discrepancy may be from the real intertube Coulomb interaction being weaker than in Ref. 8, where SWNTs with the same chirality were ideally arrayed.

Due to the fact that these peaks were observed at almost the same positions regardless of SWNT diameter and preparation method, they are attributed to the maxima in $\operatorname{Im}\left\{\varepsilon_{\perp}\right\}(\sim 4.5 \mathrm{eV})$ and $\operatorname{Im}\left\{-\varepsilon_{\|}^{-1}\right\}$ $(\sim 5.25 \mathrm{eV}$ ) reflecting optical properties of graphite. These results imply the important possibility of investigating unresolved optical properties of graphite in the direction parallel to the $c$-axis by optical absorption measurements of aligned SWNT films.

Part of this work was financially supported by KAKENHI \#16360098 and \#1610754 from JSPS, and by KAKENHI \#13GS0019 from MEXT.

\section{References}

1. S. Iijima and T. Ichihashi, Nature 363, 60 (1993).

2. R. Saito, G. Dresselhaus, and M.S. Dresselhaus, Physical Properties of Carbon Nanotubes (Imperial College Press, London, 1998).

3. R. Martel et al., Appl. Phys. Lett. 73, 2447 (1998).

4. Y. -C. Chen et al., Appl. Phys. Lett. 81, 975 (2002); Y. Sakakibara et al., Jpn. J. Appl. Phys. 42, L494 (2003); S. Yamashita et al., Opt. Lett. 29, 1581 (2004).

5. J. A. Misewich et al., Science 300, 783 (2003).

6. M. Freitag et al., Nano Lett. 3, 1067 (2003).

7. H. Ajiki and T. Ando, Physica B 201, 349 (1994).

8. M. F. Lin and D. S. Chuu, Phys. Rev. B 57, 10183 (1998); F. L. Shyu and M. F. Lin, Phys. Rev. B 60, 14434 (1999).

9. J. Hwang et al., Phys. Rev. B 62, 13310 (2000); M. Ichida et al., Appl. Phys. A 78, 1117, (2004).

10. M. F. Islam et al., Phys. Rev. Lett. 93, 37404 (2004).

11. Y. Murakami et al., Chem. Phys. Lett. 385, 298 (2004).

12. S. Maruyama et al., Chem. Phys. Lett. 360, 229 (2002).

13. Y. Murakami et al. (to be published). 
14. M. S. Strano et al., Nano Lett. 3, 1091 (2003); R. B. Weisman and S. M. Bachilo, Nano Lett. 3, 1235 (2003); S. K. Doorn et al., Appl. Phys. A 78, 1147 (2004).

15. H. Kataura et al., Synth. Met. 103, 2555 (1999).

16. T. Pichler et al., Phys. Rev. Lett. 80, 4729 (1998).

17. B. W. Reed and M. Sarikaya, Phys. Rev. B 64, 195404 (2001).

18. R. C. Haddon, J. Sippel, A. G. Rinzler, and F. Papadimitrakopoulos, MRS Bullet. 29, 252 (2004).

19. E. A. Taft and H. R. Philipp, Phys. Rev. 138, A197 (1965); M. K. Kelly et al., Phys. Rev. B 46, 4963 (1992).

20. A. Borgheshi and G. Guizzetti, in Handbook of Optical Constants of Solids II, edited by E. D. Palik (Academic Press Inc., San Diego, 1991).

21. F. J. Garcia-Vidal and J. M. Pitarke, Eur. Phys. J. B 22, 257 (2001).

22. A. G. Marinopoulos et al., Phys. Rev. Lett. 89, 076402 (2002).

23. A. B. Djurisic and E. H. Li, J. Appl. Phys. 85, 7404 (1999);

24. H. Venghaus, Phys. Stat. Solidi B 71, 609 (1975).

25. Y. Kim et al. Jpn. J. Appl. Phys. 42 (2003) 7629.

26. J. R. Lakowicz, Principles of Fluorescence Spectroscopy, $2^{\text {nd }}$ Ed. (Plenum Publishing Corp., New York, 1999).

27. H. Lee et al., Jpn. J. Appl. Phys. 42, 5880 (2003).

28. We defined the molar cross-section $\eta$ based on the definition of absorbance $\Lambda=-\log _{10} T=\eta \cdot n \cdot l$ where $T, n, l$ are the optical transmission, molar concentration of $\mathrm{C}$, and film thickness, respectively.

29. A. Grüneis et al., Phys. Rev. B 67, 165402 (2003).

30. A. Jorio et al., Phys. Rev. B 65, 121402 (2002).

31. Y. Murakami, S. Chiashi, E. Einarsson, and S. Maruyama, Phys. Rev. B 71, 085403 (2005). 


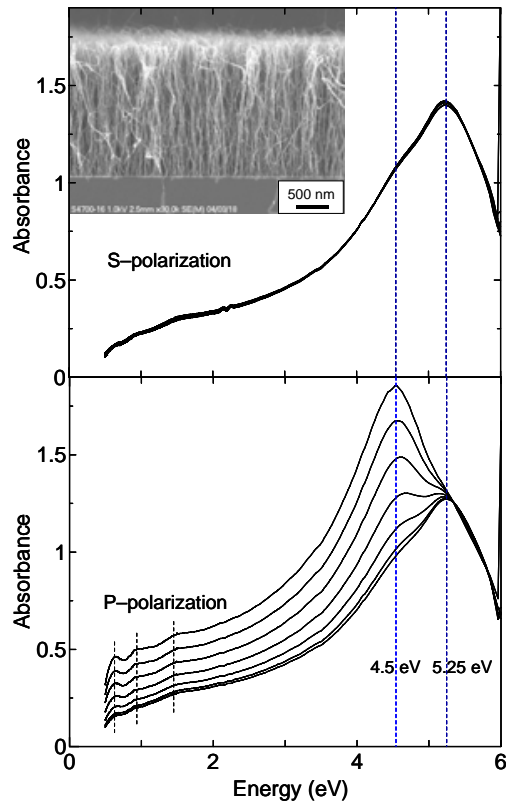

FIG. 1. Absorption spectra measured from a vertically aligned SWNT film. The incident angle $\theta$ was varied from $0^{\circ}$ (bottom spectrum) to $45^{\circ}$ (topmost spectrum) at a step of $7.5^{\circ}$. Polarizations are 's' (upper panel) and 'p' (lower panel) toward the substrate plane. Inset shows a cross-sectional FE-SEM image of the measured SWNT film.

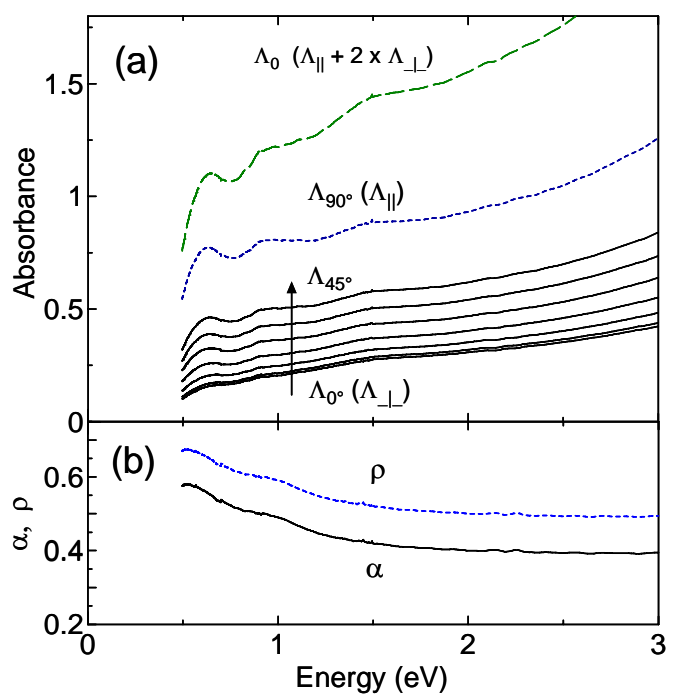

FIG. 2. (a) Magnification of lower panel of Fig. 1 for $0.5-3 \mathrm{eV}$. Solid lines are measured spectra while dotted and dashed lines denote extrapolated spectra for $\theta=90^{\circ}\left(\Lambda_{\|}\right)$and total absorbance $\left(\Lambda_{0}\right)$, respectively. (b) Calculated absorption anisotropy $(\alpha)$ and polarization $(\rho)$. 


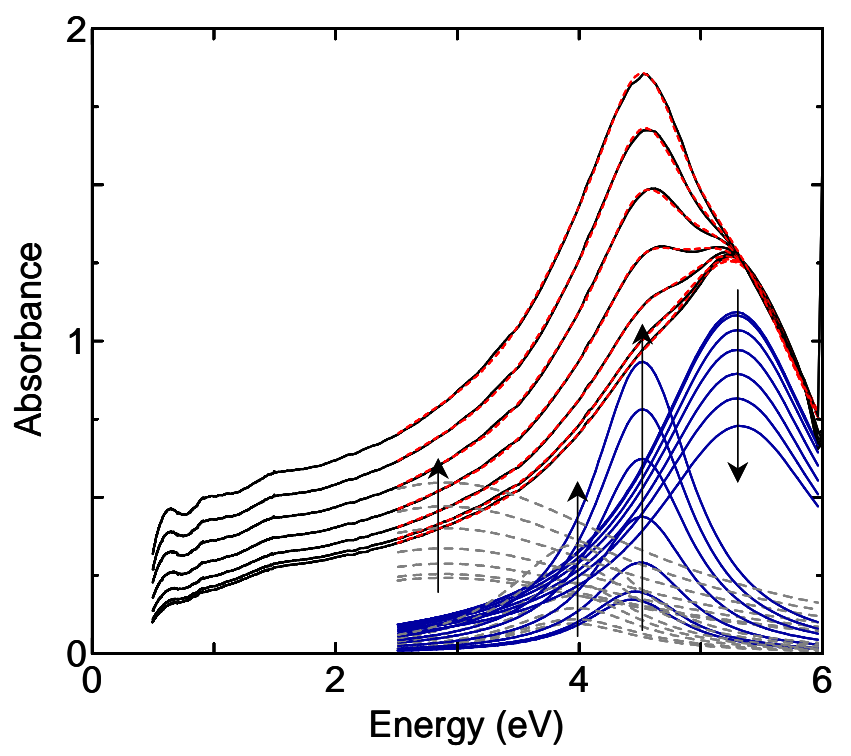

FIG. 3. (Color online) Fitting of absorption spectra shown in the lower panel of Fig. 1 by four Lorentzian curves. Arrows indicate the change of each curve as $\theta$ changes from $0^{\circ}$ to $45^{\circ}$. Red dotted lines represent the sum of the four curves.
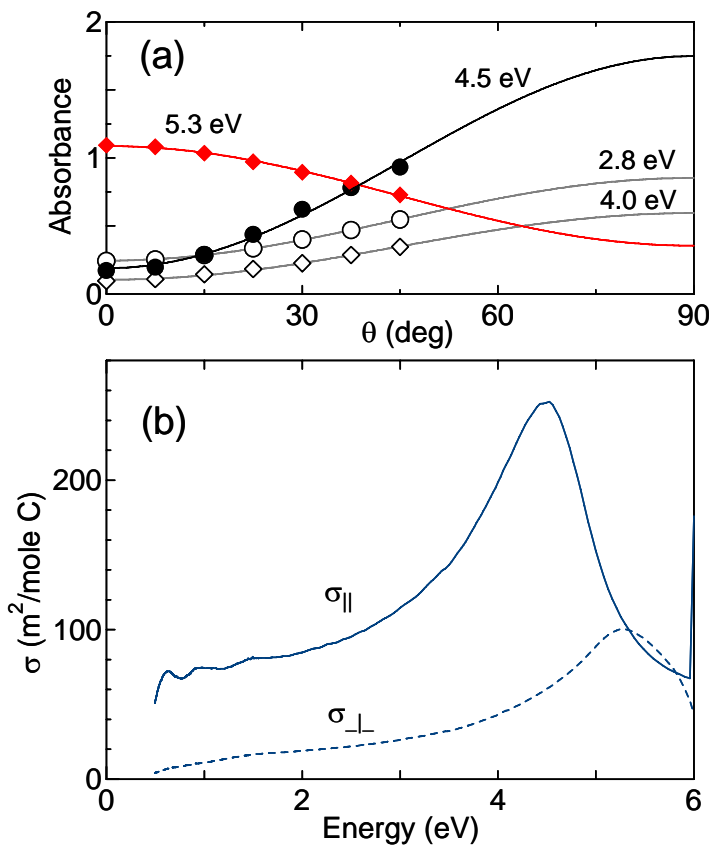

FIG. 4. (a) Dependence of each Lorentzian amplitude on $\theta$. Curves fitted by $\sin ^{2} \theta$ and $\cos ^{2} \theta$ are also shown. (b) Calculated bare optical cross-sections parallel to $\left(\sigma_{\|}\right)$and perpendicular to $\left(\sigma_{\perp}\right)$ the SWNT axis. 